\title{
LA ALFABETIZACIÓN DIGITAL DEL ALUMNADO QUE ACCEDE A LA UNIVERSIDAD DE CÓRDOBA
}

\section{DIGITAL LITERACY OF NEW STUDENTS AT THE UNIVERSITY OF CORDOBA}

\author{
Verónica Marín Díaz; vmarin@uco.es \\ Facultad de Ciencias de la Educación. Universidad de Córdoba. \\ Eloísa Reche Urbano; fe1reure@uco.es \\ Escuela Universitaria de Magisterio Sagrado Corazón. \\ Centro adscrito a la Universidad de Córdoba.
}

\begin{abstract}
RESUMEN
El objetivo prioritario del estudio que aquí se expone, se centra, a partir de las apreciaciones de los propios estudiantes, en definir el nivel de conocimiento y manejo de las herramientas tecnológicas del alumnado de nuevo ingreso de la Escuela Universitaria de Magisterio de la Universidad de Córdoba, que lo capacitan para el desempeño de la profesión y que se muestran como un elemento determinante para conseguir los objetivos del nuevo modelo educativo derivado del Espacio Europeo de Educación Superior. Para ello se ha utilizando un diseño de investigación empírica no experimental (ex post facto), descriptiva y correlacional, utilizando el estudio de encuesta, implementadas desde el año académico 2006-2007 hasta el 2009-2010 y analizadas mediante un procedimiento cuantitativo.
\end{abstract}

PALABRAS CLAVE: Enseñanza superior, Alumnado universitario, herramientas tic

\section{ABSTRACT}

The primary objective of the study presented here, focuses, from the findings of the students themselves, to define the level of knowledge and use of technological tools of new students entering the School of Education at the University of Cordoba, which enable him to carry out the profession and shown as a decisive factor in achieving the objectives of the new educational model derived from the European Higher Education Area. For it has been using an empirical research design experimental (ex post facto), descriptive and correlational study using survey, implemented since the academic year 2006-2007 to 2009-2010 and analyzed using a quantitative procedure.

KEY WORDS: Higher education, university student, ITC tools 


\section{1.- INTRODUCCIÓN}

La tecnología comenzó siendo un campo acotado para un grupo reducido de personas que bien pertenecían a una élite social o a sectores empresariales muy concretos. Esta realidad se ha visto modificada con el paso del tiempo fundamentalmente por un lado por los cambios continuos en el campo de las telecomunicaciones y por otro, por la evolución de los medios audiovisuales. En consecuencia, el avance experimentado en la última década ha derivado en un modelo social vertebrado por el desarrollo tecnológico, donde el ciudadano se ha convertido en un sujeto activo-participativo, independientemente de su estatus social.

En esta etapa, denominada por Castell (2000) era digital, la obtención de información, la creación de conocimiento y la transmisión del mismo se convierten en los elementos esenciales del crecimiento tanto de las sociedades en general como de los individuos en particular, llegando incluso hasta el extremo de modificar las conductas sociales; todo ello impulsado por la idea de un mundo global. Los cambios operados en las nuevas formas de interacción han llevado también a realizar cambios substanciales en la organización y reorganización de nuestros procesos cognitivos y prácticos, además de los sociales. En tal escenario, se aprecia un nuevo concepto conocido como alfabetización digital.

Como se refleja en la Declaración de Principios de la Cumbre Mundial sobre la Sociedad de la Información celebrada en Túnez (2004), "cada persona debería tener la posibilidad de adquirir las competencias y los conocimientos necesarios para comprender la Sociedad de la Información y la economía del conocimiento, participar activamente en ellas y aprovechar plenamente sus beneficios" (2004, p.5). Esta perspectiva integra una visión de la alfabetización en función de la adquisición de destrezas básicas, pero cabría concretar si esas habilidades se establecen en el marco del dominio de las herramientas o va más allá. Es evidente, ante este panorama, la gran importancia que cobra el acceso y la disponibilidad de las tecnologías de la información y la comunicación (TIC) y la necesidad de adquisición de competencias necesarias para saber manejarlas, pero también se hace imprescindible la competencia para la búsqueda de información o documentación, la selección, discriminación, utilización y transmisión de dicha información de manera responsable y ética. En consecuencia, habría que ser conscientes de que vivimos en un mundo que evoluciona de forma vertiginosa, siendo una de sus principales características la rápida caducidad de los mensajes, derivado este fenómeno de la producción continua y fluida de información, en virtud de ello, se debe contemplar y entender la alfabetización como "una competencia comunicativa y social que toma en cuenta el contexto de comunicación, cultural e interpersonal en el que se produce" (Rodríguez, 2004, p.432).

El desarrollo continuo de las TIC y, en consecuencia, la rápida obsolescencia de los conocimientos, así como su influencia en todos los sectores sociales y económicos requiere de profesionales con un perfil que se adapte a las constantes transformaciones y que además posea una mentalidad abierta al aprendizaje. Reconocemos que los jóvenes constituyen la fuerza de trabajo del futuro, son los principales creadores de las TIC y también los primeros que las adoptan. En consecuencia, deben fomentarse sus capacidades como estudiantes, desarrolladores, contribuyentes, empresarios y encargados de la adopción toma de decisiones (Declaración de Principios de la Cumbre Mundial sobre la Sociedad de la Información, 2004, p.2). En este sentido, los centros educativos son responsables de tratar la 
alfabetización en TIC, desde los niveles iniciales hasta los superiores, y es en este último donde se centra este trabajo. El desarrollo de los planes de alfabetización digital de las etapas de educación infantil, primaria y secundaria va a depender, en gran medida de la formación que los docentes que imparten clase en dichos niveles hayan recibido en los centros universitarios. En concreto para las dos primeras, la que han recibido en las Facultades de Ciencias de la Educación. Y para que estos profesionales de la educación reciban esos conocimientos o nociones es necesario saber qué aspectos conocen y desconocen en materia de TIC. Todo ello, además, ha de ser tenido en cuenta dentro del nuevo paradigma formativo que dibuja el Espacio Europeo de Educación Superior, en el cual se presenta al alumnado como el protagonista de su aprendizaje, con el objetivo de adquirir y desarrollar competencias orientadas a su perfil profesional.

El nuevo modelo formativo de enseñanza/aprendizaje implica, en consecuencia, pasar de enseñar a aprender al aprender a aprender (Cáceres, 2006). Así, De Miguel (2005) pone de manifiesto que: Frente al paradigma tradicional que centra el eje de la enseñanza sobre la tarea del profesor, el Espacio Europeo de Educación Superior promueve una teoría basada en el supuesto de que sólo se logra un aprendizaje eficaz cuando es el propio alumno el que asume la responsabilidad en la organización y desarrollo de su trabajo académico (p.16).

El desarrollo eficaz y con garantía de calidad de la actividad académica del alumnado en los Centros Universitarios, en el marco de las experiencias piloto de adaptación al crédito ECTS y de cara a la implementación de los planes de estudios para la obtención de las Titulaciones universitarias oficiales de grado, se ha sustentado en el uso de las TIC. Por lo que, como se ha señalado anteriormente, es necesario que el alumnado tenga unas competencias básicas en el manejo de estas herramientas que les facilite o favorezcan el desarrollo de su profesionalización, manteniendo, como se ha publicado en estudios anteriores (Marín, González y Gómez, 2007) que la carencia de estas destrezas puede condicionar su uso.

\section{2.- CARACTERÍSTICAS BÁSICAS DEL ESTUDIO}

Planteado el problema a investigar, se diseñaron como objetivos: el describir el grado destrezas en el manejo de las TIC que posee el alumnado universitario de nuevo ingreso que accede a la Escuela Universitaria de Magisterio, conocer la valoración que hace el alumnado universitario de la utilización didáctica de las TIC en el aula, así como valorar los efectos de las TIC en la práctica docente y en los procesos de enseñanza-aprendizaje, tanto en la actualidad como potencialmente con el nuevo sistema de créditos europeos, a través de la opinión de los estudiantes.

Este estudio responde a un diseño de investigación empírica no experimental (ex post facto), descriptivo y correlacional, utilizando estudios de encuestas. Para llevar lo a cabo, el desarrollo del mismo se centró en cuatro fases diferenciadas:

- Primera fase: Análisis la población y selección de la muestra.

- Segunda fase: Instrumentos de recogida de información.

- Tercera fase: Tratamiento de los datos.

- Cuarta fase: Análisis de los datos obtenidos, discusión y conclusiones.

\section{1.- La muestra}


La población estudiada fue el alumnado de nuevo ingreso de las titulaciones de la Diplomatura de Magisterio que se imparten en la Escuela Universitaria de Magisterio Sagrado Corazón, centro adscrito a la Universidad de Córdoba, desde el año académico 2006-2007 hasta el 2009-2010. Como se puede observar en la tabla 1.1, la muestra estuvo conformada por un total de 709 estudiantes, de los 907 matriculados en los cuatro años en los que se realizó el estudio, 173 pertenecientes al año académico 2006-2007, 165 del curso 2007-2006, 186 en el curso académico 2008-2009 y 185 en el curso 2009-2010, por lo que supone la opinión de un porcentaje significativo de estudiantes matriculados en los respectivos años académicos.

\begin{tabular}{|lccc|}
\hline Curso & $f$ (población) & $\mathbf{f}$ (muestra) & Representatividad \\
$2006-2007$ & 206 & 173 & $83,98 \%$ \\
$2007-2008$ & 205 & 165 & $80,48 \%$ \\
$2008-2009$ & 237 & 186 & $78,48 \%$ \\
$2009-2010$ & 259 & 185 & $71,42 \%$ \\
Total & 907 & $\mathbf{7 0 9}$ & $\mathbf{7 8 , 1 7 \%}$ \\
\hline
\end{tabular}

Tabla 1.1: Representatividad de las encuestas recogidas en función del año académico.

El alumnado que constituía la muestra quedó distribuido de las siguiente manera: 174 estudiantes a la especialidad de Educación Infantil, 135 a maestro de Educación Primaria, 158 a maestro de Educación Física, 55 a maestro Educación Musical, 63 a maestro de Lengua Extranjera y 124 a maestro de en Audición y Lenguaje, como indica la gráfica 1.1.

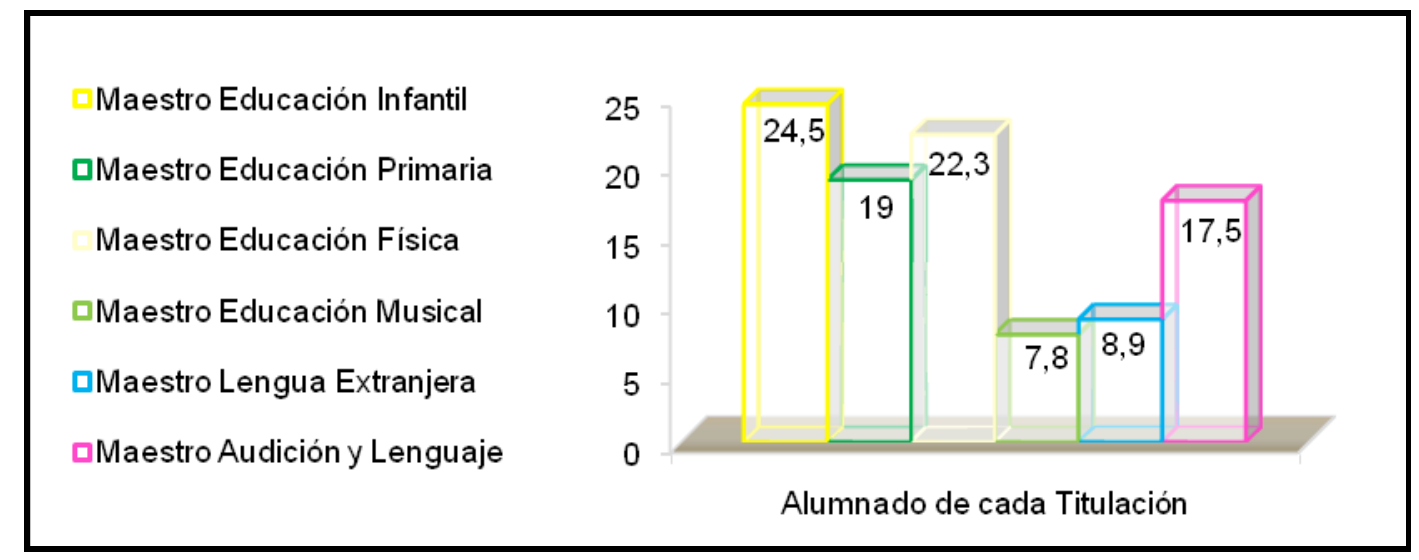

Gráfica 1.1: Distribución del alumnado en función de la titulación.

Como se puede observar en la gráfica 1.2, el 67,6\% del alumnado encuestado eran mujeres y el 32,4\% hombres, siendo la edad de la mayoría de entre los 18 a 19 años (59,2\%) y superando éstas edades el $40,8 \%$ restante (ver gráfica 1.3 ). 


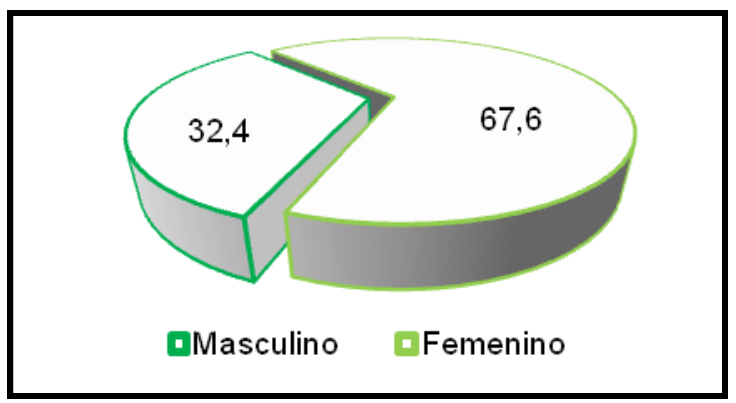

Gráfica 1.2: Distribución del alumnado en función del sexo.

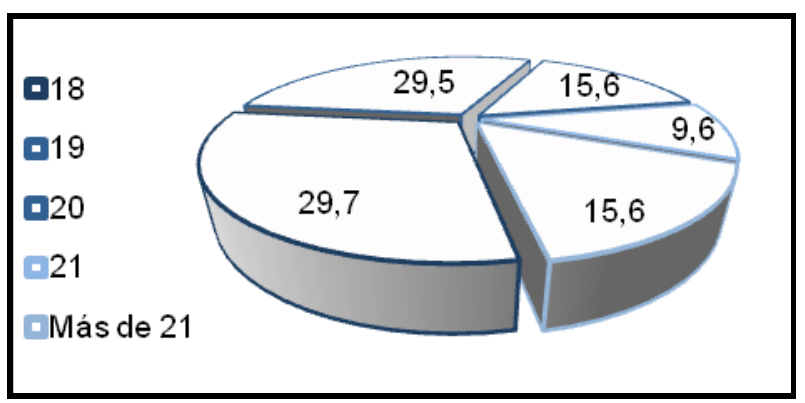

Gráfica 1.3: Distribución del alumnado en función de la edad.

Por otra parte, en relación a la dotación informática el 92,3\% del alumnado dispone de un ordenador en el lugar donde residen, siendo los y las que carecen minoría, el 7,7. De la misma manera, una amplia cifra de estudiantes cuentan con conexión a Internet, el 91,9\%, no disfrutando de ello un $8,1 \%$ de la muestra, como se puede observar en la gráfica 1.4.

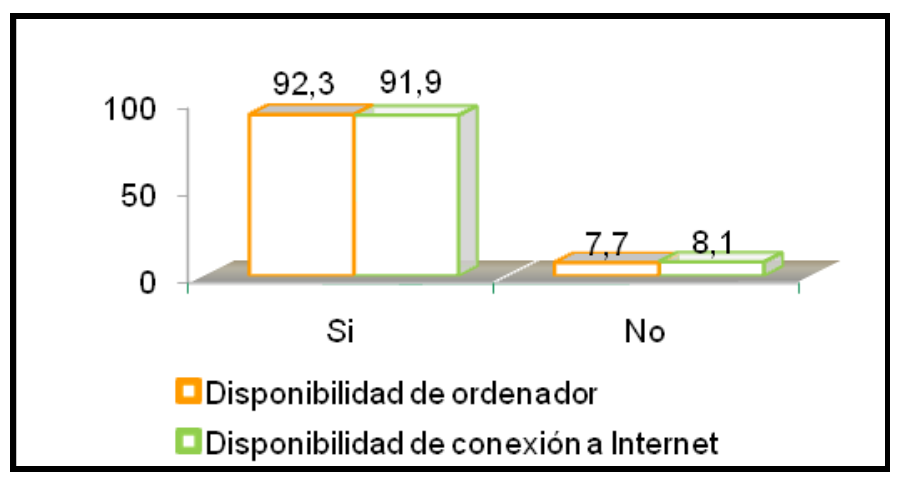

Gráfica 1.4: Distribución del alumnado en función de la disponibilidad de ordenador y conexión a Internet.

Con respecto a la formación inicial del alumnado en cetros TIC, el 39,7\% de la muestra si ha estudiado en este tipo de centros mientras que el $60,3 \%$ ha cursado sus estudios en instituciones sin éste proyecto.

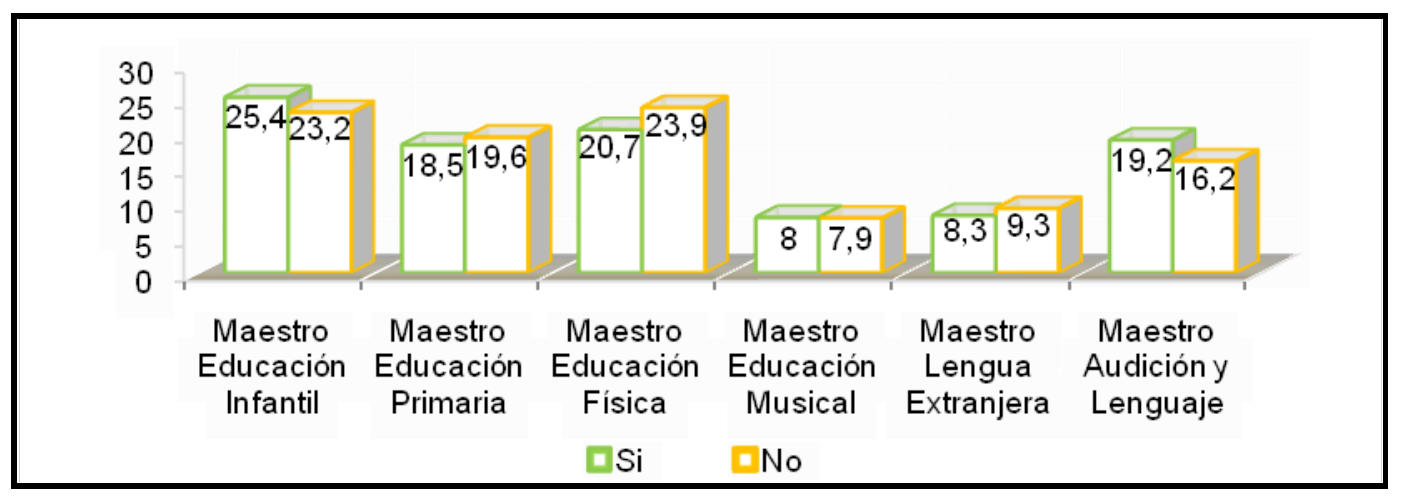




\section{2.- Instrumentos de recogida de información.}

Para confeccionar el instrumento que proporcionara respuestas al estudio planteado, se diseño un cuestionario cuyo punto de partida fue la revisión de instrumentos ya existentes relacionados con el tema. Con la ayuda de las encuestas encontradas y los objetivos planteados, se realizó la identificación y articulación de las dimensiones del estudio, determinando los siguientes bloques que se muestran en la tabla 1.3.

La primera dimensión recoge la valoración sobre el nivel de manejo de acciones básicas de sistemas informáticos, de herramientas, de programas y el uso del sistema operativo al comenzar el curso. Los bloques que la conforman, abarcan en primer lugar el nivel de manejo de acciones informáticas y sistema operativo y en segundo el nivel de manejo de herramientas y programas. Para este conjunto de ítems, los niveles que se propusieron al alumnado van desde la opción "Ninguno", "Bajo", "Medio", Avanzado" o "Experto".

La segunda dimensión comprende las valoraciones sobre los conocimientos de terminología de Internet, búsqueda y selección de información en la red, comunicación sincrónica y asincrónica, trabajo cooperativo en redes y la telegestión. Los bloques en la que se distribuyen comienzan con las estimaciones sobre la comprensión de terminología relacionada con Internet y finalizan con el nivel de manejo de acciones telemáticas. Para los ítems del primer bloque, el alumnado debía señalar aquel concepto cuyo significado entendiera y para aquellos que conformaron el segundo bloque, la opciones fueron "Ninguno", "Bajo", "Medio", Avanzado" o "Experto".

La tercera de ellas se ocupa de la aptitud y actitud ante las TIC, quedando distribuida en dos bloques, el primero de ellos refleja la identificación del alumnado con sus actitudes ante las TIC y el segundo, relativo a la apreciación y aptitud del alumnado ante las TIC en la actividad académica. En el primer bloque, las valoraciones que se les planteó al alumnado van desde la opción "Nada", "Poco", "Moderado", "Mucho" hasta la posible elección de "Bastante" y para el segundo bloque formado por dos grupos de ítems las opciones de respuesta en el primer caso van desde estar "Totalmente disconforme", "En disconformidad", "Indiferente", "De acuerdo" a un "Totalmente de acuerdo" y en el segundo conjunto de variables fueron "Nada", "Poco", "Suficiente", "Bastante" y "Mucho".

\begin{tabular}{|c|c|c|}
\hline 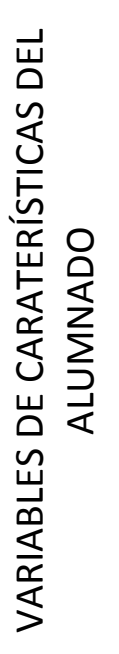 & $\begin{array}{l}\text { Variables de identificación del } \\
\text { alumnado. } \\
\text { Variables académicas. } \\
\text { Variables académicas anteriores al } \\
\text { acceso a la Universidad. } \\
\text { Variables socio-económicas y } \\
\text { familiares. } \\
\text { Variables de motivacionales. }\end{array}$ & $\begin{array}{l}\text { - Sexo. } \\
\text { - Edad. } \\
\text { - Curso académico. } \\
\text { - Titulación. } \\
\text { - Titulación más alta que poseen. } \\
\text { - Tipología de Centro donde se } \\
\text { realizaron los primeros estudios. } \\
\text { - Lugar de residencia } \\
\text { - Simultaneidad de estudios con } \\
\text { trabajo. } \\
\text { - Nivel socio-económico familiar. } \\
\text { - Motivación al elegir la carrera. } \\
\text { - Motivación al elegir la Titulación. }\end{array}$ \\
\hline
\end{tabular}




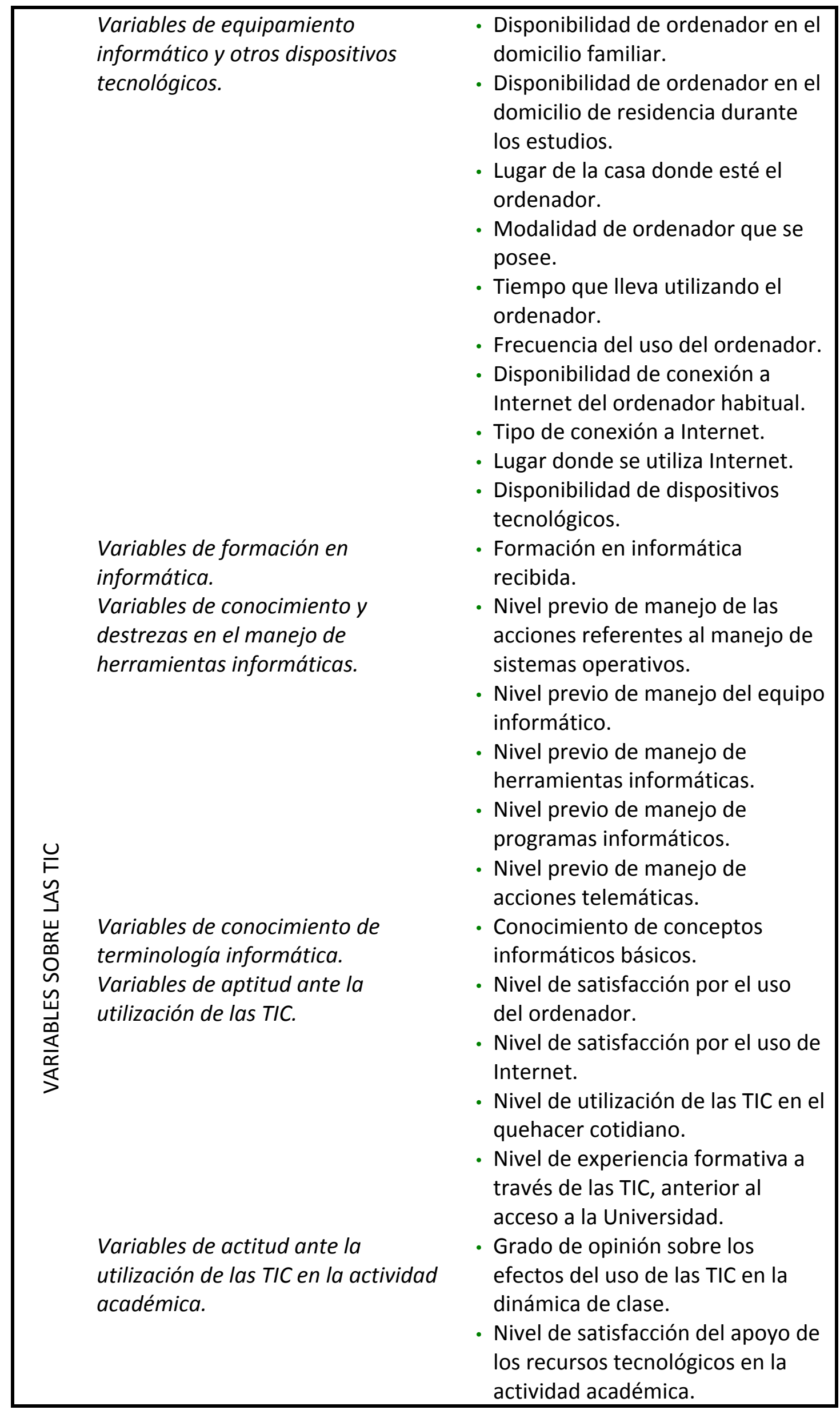

Tabla 1.3: Variables de estudio del alumnado. 
Para el estudio de la fiabilidad y validez del cuestionario se realizaron los siguientes procedimientos; tras una primera aplicación experimental a los estudiantes de nuevo ingreso del curso 2006-2007, con el objeto de generar el instrumento definitivo, se volvió a administrar al alumnado de nuevo ingreso de los cursos 2007-2008, 2008-2009 y 2009-2010 y se realizaron los siguientes análisis:

Análisis de Consistencia Interna, en el sentido de dotar de significación a los ítems de la prueba. Para ello se utilizó el coeficiente Alfa de Cronbach (Cronbach, 1951), cuyo valor para el total del cuestionario fue de 0,93 , indicando un nivel elevado de estabilidad en las respuestas, por lo que el cuestionario presentó indicios de garantías de fiabilidad.

- Análisis de la capacidad de discriminación de los elementos de modo que se refuerce el carácter unidimensional de la prueba (García, Gil y Rodríguez, 1995, p. 24). Se utilizó para ello la t de Student entre las medias de los grupos establecidos para los 87 ítems cerrados de elección ordinales (escala de valoración de 1 a 5). En este aspecto, todos los ítems a excepción de cinco, resultaron tener un elevado poder de discriminación, reflejando la existencia de una estructura interna en el cuestionario capaz de responder a las demandas planteadas.

Validez interjueces para buscar la representatividad o adecuación del instrumento al tema objeto de estudio. Dicha comprobación del contenido del instrumento se llevo a cabo de la mano de 5 jueces: 3 especialistas en el Área de Didáctica, de las Titulaciones de Magisterio, de las Facultades de Ciencias de la Educación de las Universidades de Cádiz, Granada y Sevilla y 2 especialistas en el Área de Métodos de Investigación y Diagnóstico en Educación, de la Licenciatura de Psicopedagogía de la Universidad de Córdoba. Se mantuvieron sesiones de asesoría on-line, con los jueces, en torno a las sugerencias y modificaciones que proponían.

- Análisis factorial para evidenciar la presencia de validez del instrumento mediante el análisis estructural de covarianza de los ítems (Kerlinger, 2002). En este proceso se inició el análisis tras establecer los métodos de extracción (componentes principales) y de rotación (varimax), seleccionando las variables que hacen referencia a las cuestiones planteadas, obteniendo 10 factores, con una explicación total de la varianza del 59,797\%. Al grupo de ítems que los definen, se le realizó el estudio de consistencia, con valores Alfa superiores a 0.77 , considerándose fiables los factores derivados del análisis.

Para finalizar, se confeccionó un documento informativo dirigido al alumnado, donde se presentaron las finalidades del cuestionario, solicitándoles su colaboración y la máxima sinceridad en las respuestas, comentándole que no hay respuestas correctas o incorrectas, sólo la particularidad de cada individuo. En él, también quedaba indicado el carácter anónimo del mismo.

\section{3.- Tratamiento de los datos.}

El tratamiento de los datos cuantitativos se realizó con el programa SPSS, versión 18.0, y se extrajeron los estadísticos descriptivos básicos de cada uno de los ítems: frecuencias, porcentajes, así como medidas de tendencia central para las opciones de carácter escalar. A continuación, para los ítems con opción de respuesta de relleno o escalar se efectuó un estudio correlacional entre variables a través del análisis de la varianza (ANOVA), comprobando así, la existencia o no, de diferencias significativas entre el alumnado, evidenciadas con la prueba post-hoc HSD de Tukey. 
Tras las pruebas practicadas se seleccionaron aquellas que presentaron correlaciones apreciables, las cuales en la mayoría de las dimensiones fueron con los ítems años académicos y especialidades. También se llevo a cabo una prueba de significación t Student en función de la variable sexo.

Para finalizar, se llevó a cabo la prueba no paramétrica ji cuadrado con el grupo de ítems de opción de respuesta dicotómica comprensión de la terminología relacionada con la red, resultando significativos los resultados en función de las variables años académicos, especialidades y sexo.

\section{3.- Resultados iniciales}

Teniendo en cuenta la importancia que tiene para la actividad académica del alumnado universitario, el conocer y manejar las herramientas tecnológicas básicas, así como el papel relevante de la telemática, los datos más destacados de este estudio se sitúan en torno al nivel de manejo que afirman poseer el alumnado al respecto sobre estas cuestiones.

El primer grupo de variables analizadas hacen referencia al manejo del entorno de los sistemas operativos, tanto de aparatos o dispositivos auxiliares, como en acciones de manteniendo y optimización de su rendimiento y los resultados obtenidos reflejan que en general el alumnado de nuevo ingreso tiene una habilidad media en la mayoría de las acciones básicas, ubicándose algunos de los valores por encima de la media, como se muestra la gráfica 1.5, sobre todo el dominio de la terminología básica del sistema operativo $(\bar{x}=3,97)$ y guardar y recuperar información de diferentes soportes $(\bar{x}=3,65)$. Sin embargo, se consideran menos diestros en realizar actividades básicas de mantenimiento $(\bar{x}=2,79)$, al igual que en el uso de utilidades propias del sistema operativo $(\bar{x}=2,94)$.

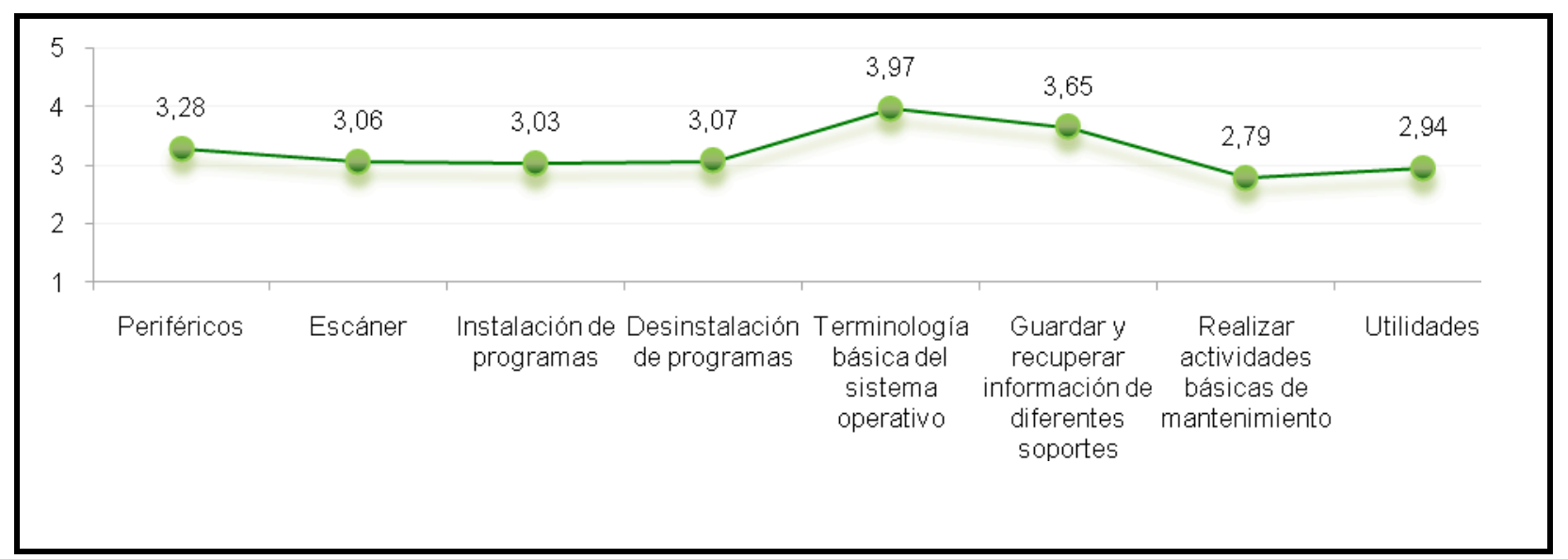

Gráfica 1.5: Media del nivel de manejo de acciones básicas de sistemas informáticos.

Con respecto al conjunto de variables que hacen referencia al manejo de herramientas y programas informáticos, como se puede apreciar en la gráfica 1.6, el alumnado posee unas destrezas reducidas en la mayoría de los casos, destacando con valores por encima del nivel medio, los programas para procesar textos $(\bar{x}=3,32)$ y para realizar presentaciones $(\bar{x}=3,14)$.

Por el contrario, llama la atención por ser una de las herramientas consolidadas en la práctica académica actual que el uso de las plataformas de enseñanza presenten datos de manejo tan bajos $(\bar{x}=2,27)$, al igual que ocurre para aplicaciones o programas de diseño de materiales multimedia $(\bar{x}=2,15)$, así como el software libre $(\bar{x}=2,16)$, siendo una apuesta para los centros educativos y de gran utilidad para su futura profesión. 


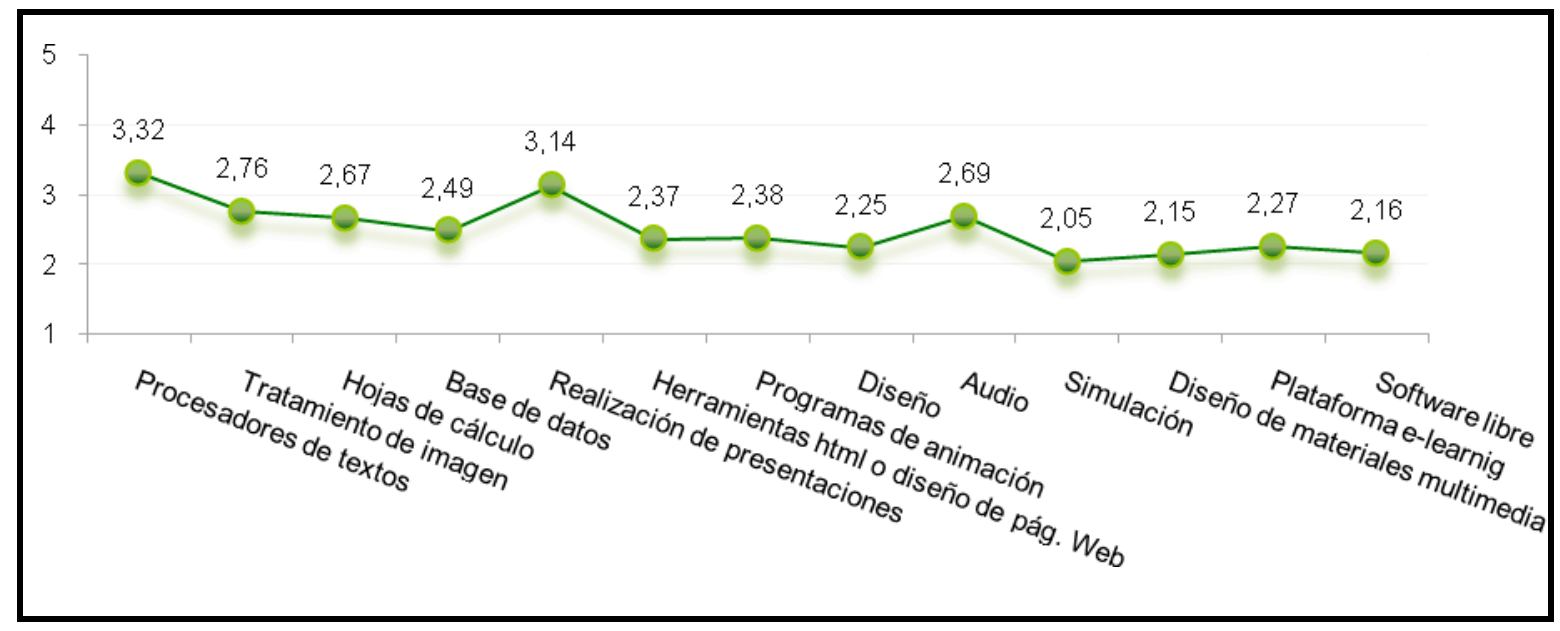

Gráfica 1.6: Media del nivel de manejo de herramientas y programas.

Por otra parte, se preguntó al alumnado sobre el conocimiento de la terminología derivada de los diferentes contextos de la red, teniendo en cuenta que Internet ha generado un vocabulario específico cada vez más rico y extendido que estará presente en el periodo de profesionalización. Tal y como se muestra en la gráfica 1.7, las expresiones más conocidas por los estudiantes son página web (99\%), portal (78,6\%), hipervínculo (69,3\%) y link (68\%) frente a los términos frame $(90,6 \%)$, hosting $(89,7 \%), \operatorname{FTP}(86,9 \%)$, banner (85,3\%), m-learnig $(84,1 \%)$ y web $2.0(78,8 \%)$ que resultan ser los que menos identifican los estudiantes encuestados.

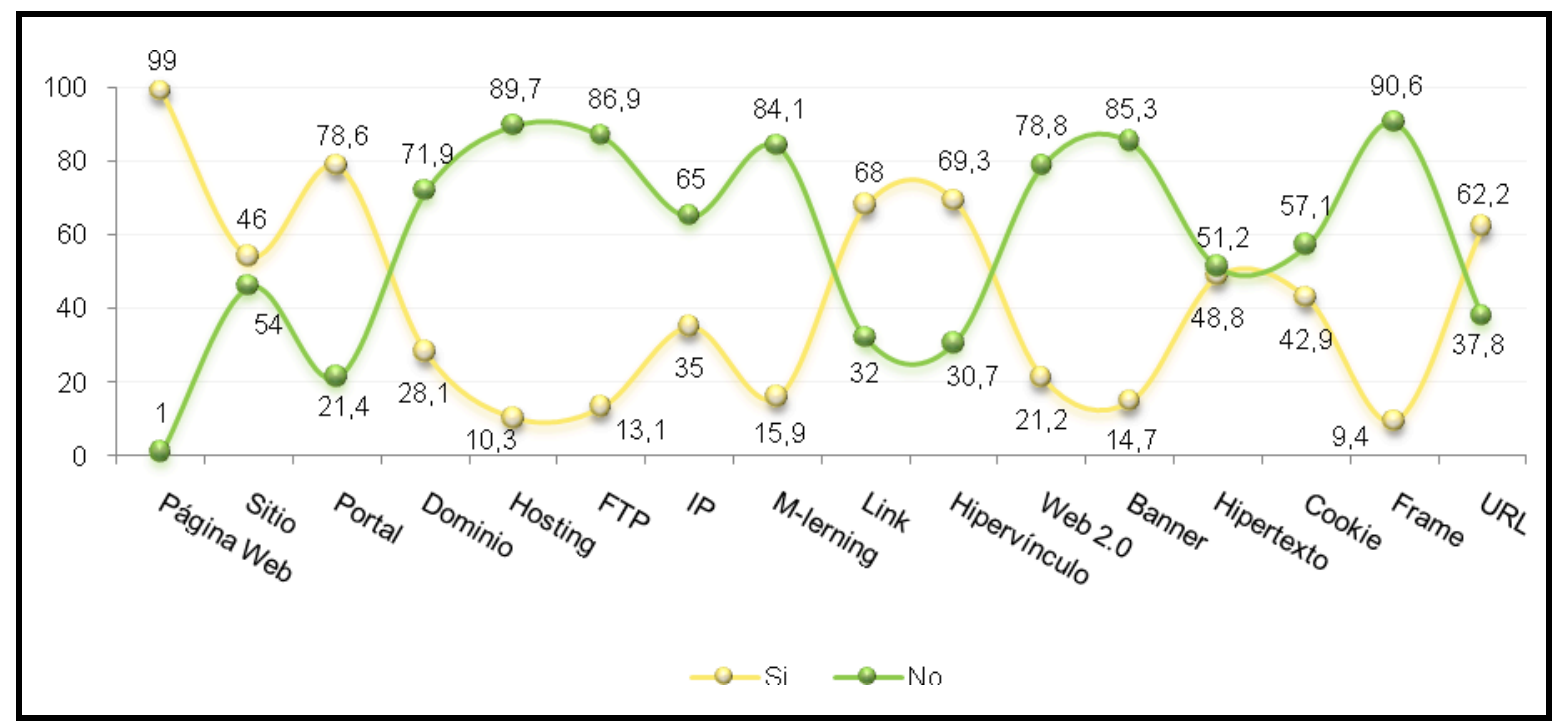

Gráfica 1.7: Distribución de los porcentajes nivel de compresión de terminología relacionada con Internet.

Por último, se exponen los datos que conformaron el grupo de variables que aluden a una representación básica de acciones telemáticas que van desde navegar por Internet y seleccionar información, pasando por el dominio de algunos sistemas de comunicación sincrónica, como por ejemplo, videoconferencia y de tipo asincrónica, como correo electrónico, con la posibilidad de adjuntar archivos. Por otro lado, también consta de ítems que se refieren al uso de aplicaciones que se engloban dentro de la tecnología Web 2.0 y que pueden ser utilizados en los procesos de E-A, como son los blog, wiki y webquest así como, diversas gestiones personales que se pueden tramitar o formalizar a través de la red y que facilitan acciones cotidianas como una consulta bancaria o una compra, sin dejar de lado la matriculación en una titulación vía Internet (automatrícula). 
A diferencia de los datos obtenidos en el resto de grupos de variables, éste presenta en general resultados más altos con valores muy por encima de la media (ver tabla 1.8), al menos en lo que se refiere a las destrezas manifestadas por el alumnado en cuanto a navegar por Internet $(\bar{x}=4,04)$, manejo del correo electrónico $(\bar{x}=4,19)$, adjuntar archivos en email $(\bar{x}=4,06)$ y chatear $(\bar{x}=4,2)$. También, el alumnado alega saber operar de manera óptima por a red $(\bar{x}=3,51)$, seleccionar información $(\bar{x}=3,81)$, así como participar en un foro $(\bar{x}=3,53)$ y en una videoconferencia $(\bar{x}=3,24)$.

Por el contrario, aquellas acciones relacionadas con tecnologías Web 2.0 ostentan datos bajos como en el caso de la creación o participación en blog $(\bar{x}=2,95)$, utilizar una webquest $(\bar{x}=2,24)$ y crear o consultar una wiki $(\bar{x}=2,24)$, al igual que ocurre en actividades de telegestión $(\bar{x}=1,85)$ o en la formalización de la automatrícula $(\bar{x}=2,56)$.

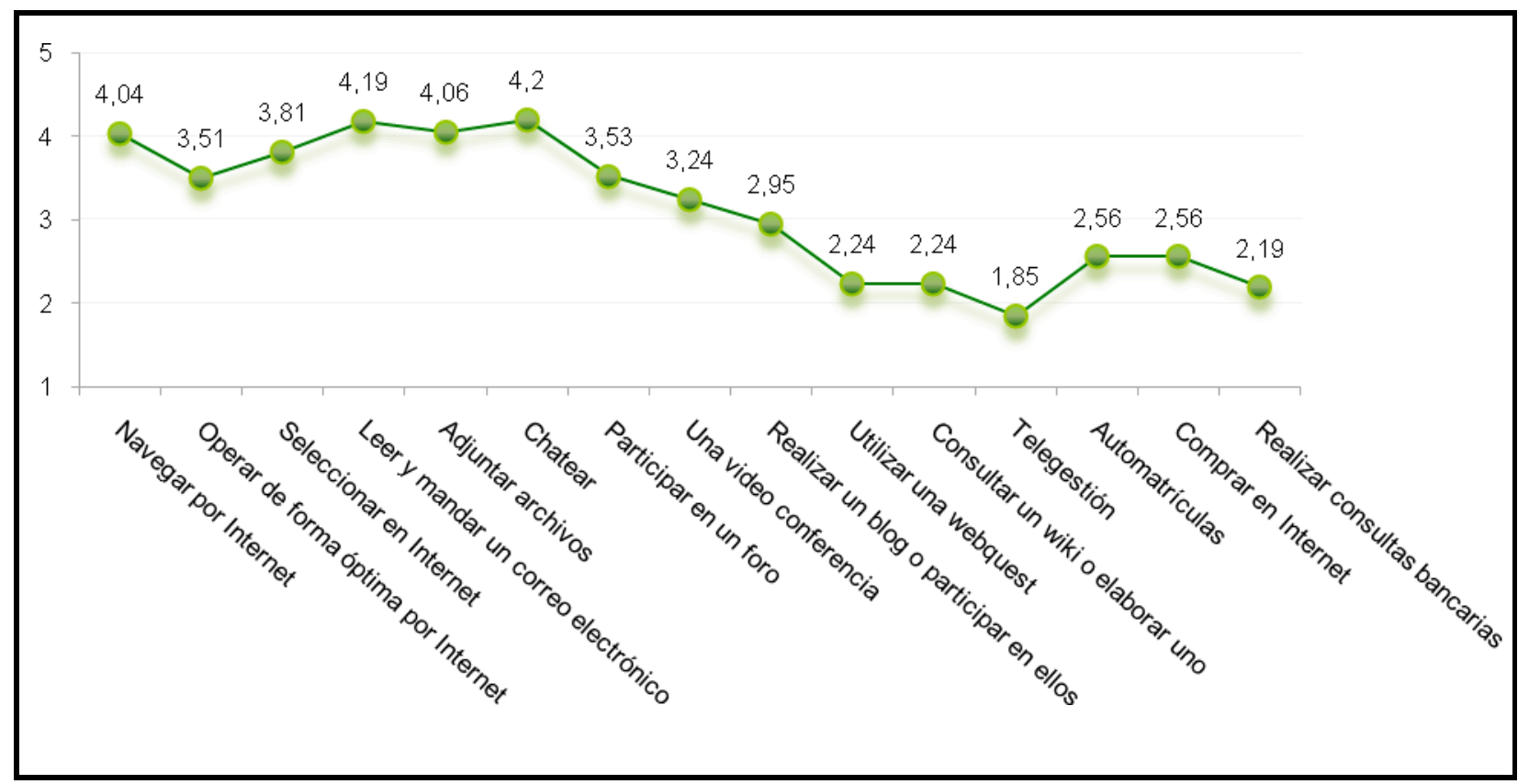

Gráfica 1.8: Media del nivel de manejo de acciones telemáticas.

\section{4.- CONCLUSIONES INICIALES}

La realización de estudios sobre el conocimiento y el uso o manejo que los estudiantes universitarios hacen de las TIC, comienzan a ser numerosos en nuestro panorama, ejemplo de ello son las publicaciones de Comas, Sureda y Mut (2010) o Henríquez-Ritchie y Organista-Sandoval (2009). De ahí que se trate de dibujar el perfil del futuro alumnado que ocupará las aulas en las que se desarrolle el EEES dentro del mundo tecnológico que presentaran las aulas en las que desarrollaran su profesión, muestra de este avance tecnológico de las aulas de infantil y primaria es el plan Escuela 2.0 que en nuestro país se ha puesto en marcha.

En este primer avance del estudio llevado a cabo, se han expuesto de manera descriptiva los resultados que dan respuesta al primer objetivo planteado, por el que se pretende precisar el grado destrezas en el manejo de las TIC del alumnado universitario de nuevo ingreso a través de su propia apreciación. A groso modo, los resultados obtenidos han revelado que el alumnado se autopercibe con unas destrezas moderadas en la selección de acciones, 
herramientas, aplicaciones y programas por las que se le preguntó, así como desconoce la mayoría de la terminología que llega de la mano de Internet, salvo aquellos más usuales.

Centrando la atención en los bloques que hacen referencia al manejo de programas informáticos y concretamente al que alude a las acciones telemáticas, llama la atención los niveles medios reflejados por los estudiantes, en el caso de herramientas para procesar textos y para la elaboración de presentaciones, así como los datos obtenidos por debajo de la media, en cuanto a las destrezas con aplicaciones para el diseño de materiales multimedia y en el manejo de la plataforma educativa. De la misma manera, aunque el alumnado se describe con un nivel aceptable en cuanto a navegar por la red, si sorprende el bajo dominio que presentan para ser operativos, así como para seleccionar información, teniendo en cuenta que, como indican Ferro, Martínez y Otero, (2009), una de las ventajas que tienen las TIC en los procesos de enseñanza-aprendizaje es la facilidad de acceso a la información y la posibilidad de interactuar con ella.

Sin embargo, habría que destacar la familiarización de estas generaciones de inmigrantes digitales con el mundo de las aplicaciones o programas que permiten comunicarse con otros usuarios, donde ellos se ven con un dominio suficientemente considerable. Este aspecto resulta positivo, pues permite establecer vías de comunicación no presencial o asincrónica, que esta comunicación sea más flexible y que aporte nuevos escenarios para el trabajo cooperativo, además de ello facilitan las tutorías virtuales, entre ostros aspectos.

Por último, cabe señalar que los resultados aquí expuestos, aunque básicos, permiten realizar una primera aproximación a las carencias formativas sobre TIC del alumnado que accede a la Universidad en los momentos actuales y que pueden condicionar la utilización eficaz y eficiente de estas herramientas en procesos pedagógicos, sin dejar a un lado que la aplicación de las TICS motiva a los alumnos y capta su atención, convirtiéndose en uno de los motores del aprendizaje ya que incita a la actividad y al pensamiento (Ferro, Martínez y Otero, 2009, p.5).

\section{REFERENCIAS BIBLIOGRÁFICAS}

CÁCERES GONZÁLEZ, P. (2006). Efectos del EEES en la actividad Académica. Evaluación y mejora de los aprendizajes de los estudiantes. Ponencia JORNADAS INFORMATIVAS SOBRE EL ESPACIO EUROPEO DE EDUCACIÓN SUPERIOR (EEES) DIRIGIDAS A LOS ESTUDIANTES DE LA UPCT. 25 al 28 de septiembre de 2006. Disponible en http://www.upct.es/contenido/conv_euro/ficheros/54Ponencia\%20Pilar\%20Caceres.pdf

CASTELLS, M. (2000). La era de la información. Economía, Sociedad y Cultura. 2a edic. Vol. 1 La Sociedad Red. Madrid: Alianza.

CRONBACH, J.L. (1951). Coefficient alpha and the internal structure of test. Psychometrika, 16, 297-334.

DECLARACIÓN DE PRINCIPIOS. CUMBRE MUNDIAL DE LA SOCIEDAD DE LA INFORMACIÓN. (2004). Túnez. Disponible en http://www.itu.int/wsis/documents/doc_multi.asp?lang=es\&id=1161|0

DE MIGUEL, M. (2005). Cambio de paradigma metodológico en la Educación Superior. Exigencias que conlleva. Disponible en http://cuadernosie.info/files/2005-CIE-02.pdf 
FERRO, C., MARTÍNEZ, A.I. y OTERO, M.C. (2009). Ventajas del uso de las tics en el proceso de enseñanza-aprendizaje desde la óptica de los docentes universitarios españoles. EDUTEC-E, Revista Electrónica de Tecnología Educativa, 29. Disponible en http://edutec.rediris.es/Revelec2/revelec29/articulos_n29_pdf/5Edutec-E_Ferro-MartinezOtero_n29.pdf

GARCÍA JIMÉNEZ, E.; GIL FLORES, J. y RODRÍGUEZ GÓMEZ, G. (1995). Introducción a la teoría clásica de los tests. Sevilla: Grupodelta.

KERLINGER, F. N. (2002). Investigación del comportamiento. Métodos de Investigación en Ciencias Sociales. (4aㅡ ed.). México, D.F.: Interamericana.

LATORRE, A.; Del RINCÓN, D. y ARNAL, J. (1996). Bases metodológicas de la investigación educativa. Barcelona: GR92.

MARÍN DÍAZ, V.; GONZÁLEZ LÓPEZ, I. y GÓMEZ PARRA, Mạ E. (2007). Assesment of the European credit system from the perspective of university students. Educational Practice and Theory, 29 (2). 49-59.

RODRÍGUEZ, J. (2004). Las alfabetizaciones digitales. Revista pedagógica BORDÓN, 56 (3 y 4), 431-441.

RITCHIE, P. y ORGANISTA-SANDOVAL, J. (2009). Definición y estimación de tipos y niveles de uso tecnológico: una aproximación a partir de estudiantes de recién ingreso a la universidad. EDUTEC-E, Revista Electrónica de Tecnología Educativa, 30. Disponible en http://edutec.rediris.es/Revelec2/revelec30/edutec30_definicion_estimacion_tipos_niveles _uso_tecnologico.html.

COMAS, R., SUREDA, J. y MUT, T. (2010). Uso de fuentes documentales -impresas y digitalescon fines académicos entre el alumnado universitario. EDUTEC-E, Revista Electrónica de Tecnología Educativa, 31. Disponible en http://edutec.rediris.es/Revelec2/revelec31/edutece31_uso_fuentes_documentales_fines_academmicos_alumnado_universitario.html.

Para citar este artículo:

MARÍN, V.; RECHE, E. (2011) «La alfabetización digital del alumnado que accede a la Universidad de Córdoba» [artículo en línea]. Edutec-e, Revista Electrónica de Tecnología Educativa. Núm. 35 / Marzo 2011. [Fecha de consulta: dd/mm/aa].

http://edutec.rediris.es/revelec2/revelec35/

ISSN 1135-9250. 